\title{
Der Einfluss des Caffeins und Theobromins auf die Aus- scheidung der Purinkörper im Harne.
}

Von

Martin Krilger und Julius Schmid.

(Ans der medicinischen Klinik der Unirersität Breslau.)

(Der Redaction zugegangen am 28. December 1900.)

Der Uebergang von Caffein in den Harn ist von Aubert ${ }^{1}$ ) beim Menschen nach Genuss von Kaffee, von Maly und Andreasch ${ }^{2}$ ) bei einem Hunde nach Caffeinfütterung beobachtet und später von E. Rost ${ }^{3}$ ) einer eingehenden Untersuchung am Menschen, Hunde und Kaninchen unterzogen worden. Dass auch das Theobromin zum Theil unverändert den Organismus passiren kann, ist von Mitscherlich, ${ }^{4}$ ) Hoffmann, 5) Rost, ${ }^{6}$ ) Krüger und Schmidt ${ }^{7}$ ) festgestellt worden.

Auf die Ausscheidung der Harnsäure hat das Caffein nach Schutzkwer ${ }^{8}$ ) und Minkowski ${ }^{9}$ ) keinen Einfluss. Wie sich Theobromin in dieser Hinsicht verhält, ist noch unbekannt. Wichtiger als jene Beobachtungen über die theilweise Beständigkeit des Caffeins und Theobromins im thierischen Stoffwechsel ist der von Albanese, ${ }^{10}$ ) Bondzyński und Gottlieb ${ }^{11}$ ) ermittelte Befund, dass diese beiden, in den Genussmitteln vorkommenden Xanthinbasen im Organismus durch

1) Pflüger's Archiv, Bd. V, S. 589.

2) Monatshefte für Chemie, Bd. IV, S. 384.

3) Archiv für exper. Pathologie u. Pharmakol., Bd. XXXVI, S. 64 .

4) Nach Rost, l. c., S. 65.

5) Archiv für exper. Pathologie u. Pharmakol., Bd. XXVIII, S. 1.

6) 1. c., S. 70 .

7) Berichte der deutschen chem. Gesellsch., Bd. XXXII, S. 2677.

8) Inaug.-Dissert., Königsberg, 1882.

9) Archiv für exper. Pathologie u. Pharmakol., Bd. XXXXI, S. 406.

10) Archiv für exper. Pathologie u. Pharmakol., Bd. XXXV, S. 447,

11) ibidem., Bd. XXXVI, S. 45. 
Beseitigung von Methylgruppen in niedere Homologe des Xanthins übergehen und somit zur Bildung von Purinbasen Veranlassung geben, welche sich gegen ammoniakalische Silberlösung und gegen Kupferoxydulsalze wie die aus dem Zerfall der Nucleine herrührenden und im Harne erscheinenden Nucleinbasen verhalten.

Geht aus dem Mitgetheilten schon mit Sicherheit hervor, dass Caffein und Theobromin eine Vermehrung der Purinbasen im Harne bewirken müssen, so liegen über die Grösse dieser Vermehrung bisher zwei Beobachtungen vor. Albanese ${ }^{1}$ ) hat nach Einnahme von $2 \mathrm{~g}$ Caffein innerhalb drei Tagen mit dem Harne 0,2 g Purinbasen ausgeschieden. Die normale Ausscheidung bei Enthaltung von Kaffee etc. betrug bei ihm innerhalb zwei Tagen $0,03 \mathrm{~g}$, innerhalb drei Tagen demnach 0,045 g. Die Einnahme von 2 g Caffein hat eine Vermehrung der Purinbasen um $0,155 \mathrm{~g}$ bewirkt.

Ferner haben Burian und Schur ${ }^{2}$ ) den Einfluss von Kaffee auf die Vermehrung des Purinbasenstickstoffes festgestellt. Sie haben sich hierbei einer modificirten Salkowski'schen Methode bedient und gefunden, dass $35-40 \%$ des Caffeinstickstoffes in Harnpurinstickstoff übergehen.

Wir haben in derselben Weise wie Burian und Schur den Einfluss des Caffeins auf die Purinbasenausscheidung durch die Bestimmung der Zunahme an Basenstickstoff ermittelt; nur haben wir statt Kaffee reines Caffein gegeben. Die von uns angewendete Methode zur Bestimmung der Harnsäure und Basen war dieselbe, wie Krüger und Schmidt ${ }^{8}$ ) zur Isolirung der Purinkörper aus Harn angewendet haben; d. h. der Harn wird siedend heiss mit Kupfersulfat und Bisulfit ausgefällt. Die aus dem Kupferniederschlage durch Natriumsulfid und Ansäuern mit Salzsäure isolirten Purinbasen werden von der nach mehrstündigem Stehen ausgeschiedenen Harnsäure durch Filtration getrennt. Nachdem der Rest der Harnsäure durch Oxydation mit Braunstein in essigsaurer Lösung zerstört ist,

1) 1. c., S. 460 .

2) Archiv für die ges. Physiol., Bd. LXXX, S. 321,

3) Berichte der deutschen chem. Gesellsch., Bd. XXXII, S. 2677. 
wird der Stickstoffgehalt der Basen in der Fällung durch das Kupferreagens bestimmt.

Der Patient, welcher sich für unsere Zwecke bereitwilligst zur Verfügung stellte, war ein 16jähriger Knabe, Fr. Fr., von 42,1 Kilo (Dystroph. muscul.).

Er hatte schon mehrere Wochen vor dem Versuche eine möglichst gleichmässige Nahrung erhalten, welche auch während des Versuches beibehalten wurde und frei von caffein- und theobrominhaltigen Genussmitteln war. Dieselbe bestand aus $250 \mathrm{~g}$ Fleisch, 2 Eiern, 1 Liter Milch, $60 \mathrm{~g}$ Butter, $200 \mathrm{~g}$ Brod und 1 Flasche Bier. In der Wahl des Fleisches wurde dem Patienten eine gewisse Freiheit gelassen, insofern er zwischen Kalbsschnitzel, Schinken, Wurst und gehacktem Rindfleisch wählen durfte.

Um die Ausscheidung an Harnsäure und Basen nach genannter Kost kennen zu lernen, wurde der Harn unseres Patienten zunächst während eines Zeitraumes von 13 Tagen einer täglichen Untersuchung auf die genannten Körper, wie auf Gesammtstickstoff unterzogen, und zwar wurden alle Werthe für Gesammtstickstoff (Ges.-N), Harnsäurestickstoff ( $\overline{\mathrm{U}} . \mathrm{N}$ ) und Basenstickstoff (B.-N) durch je 2 Analysen ermittelt, deren Mittelzahlen in Tabelle I, Seite 107, angegeben sind.

Die mittlere ${ }^{1}$ ) Ausscheidung an Harnsäure- $\mathrm{N}$ betrug demnach bei unserem Patienten 0,2220 g, an Basen-N 0,0166 g. Das Verhältniss von Gesammt-N zu Harnsäure-N ist im Mittel $53,7: 1$, das von Harnsäure-N zu Basen-N 13,5 : 1 .

Die täglichen Werthe für Harnsäure- $\mathrm{N}$ schwanken von 0,1882 bis 0,2510 g. Die Werthe für Basen- $\mathrm{N}$ bewegen sich innerhalb der Grenzen 0,0143 und $0,0188 \mathrm{~g}$. Es ist zweifellos, dass eine auch in Bezug auf die Art des Fleisches vollkommen gleichmässige Kost die Differenzen zwischen den Harnsäurewerthen herabdrücken und damit auch das Verhältniss von Harnsäure- $\mathrm{N}$ zu Basen- $\mathrm{N}$, welches bei unserem Patienten von $11,9: 1$ bis $15,1: 1$ schwankt, constanter machen würde.

1) Bei Berechnung der Mittelwerthe mussten die Zahlen vom 18./19. III. wegfallen, weil an diesem Tage ein Theil des Harnes verloren gegangen war. 
Tabelle I.

\begin{tabular}{|c|c|c|c|c|c|c|c|}
\hline Tag & $\begin{array}{l}\text { Har } \\
\text { men }\end{array}$ & & Ges.-N & $\overline{\mathrm{U}} .-\mathrm{N}$ & B. $-\mathrm{N}$ & $\frac{\text { Ges.-N }}{\overline{\mathrm{U}} .-\mathrm{N}}$ & $\frac{\overline{\mathrm{U}} \cdot-\mathrm{N}}{\text { B.-N. }}$ \\
\hline 1900 & & & & & & & \\
\hline 8.-9. III. & 550 & $\mathrm{~cm}$ & $9,24 \mathrm{~g}$ & $0,1882 \mathrm{~g}$ & $0,0143 \mathrm{~g}$ & $49,0: 1$ & $13,1: 1$ \\
\hline 9.-10. III. & 673 & $\triangleright$ & 11,17 & 0,2151 , & 0,0174 & $51,9: 1$ & $12,4: 1$ \\
\hline 10.-11. III. & 770 & $\triangleright$ & 10,23 & 0,1912 & 0,0151 & $53,5: 1$ & $12,6: 1$ \\
\hline 11.-12. III. & 842 & 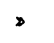 & 11,43 & 0,2058 , & 0,0150 & $55,5: 1$ & $13,7: 1$ \\
\hline 12.-13. III. & 975 & $\triangleright$ & 10,48 & 0,2183 . & 0,0150 » & $48,0: 1$ & $14,6: 1$ \\
\hline 13.-14. III. & 9555 & » & 13,97 & 0,2510 & 0,0184 & 5็5,6 : 1 & $13,6: 1$ \\
\hline 14.-15. III. & 850 & $\triangleright$ & 14,31 & 0,2413 & 0,0174 & ๖ั9,2: 1 & $13,9: 1$ \\
\hline 15.-16. III. & 800 & $\triangleright$ & $14,14=$ & 0,2274 & 0,0169 & $62,2: 1$ & $13,5: 1$ \\
\hline 16.-17. III. & 698 & $\triangleright$ & 12,56 & 0,2236 & 0,0188 & ๖ั6,1 : 1 & $11,9: 1$ \\
\hline 17.-18. III. & 675 & $\triangleright$ & 12,59 & 0,2285 & 0,0164 & $55,1: 1$ & $13,9: 1$ \\
\hline 18.-19. III. & 485 & 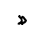 & 9,01 & 0,1433 , & 0,0128 » & $62,8: 1$ & $11,2: 1$ \\
\hline 19.-20. III. & 705 & $\triangleright$ & $11,78=$ & 0,2256 & 0,0171 & $52,7: 1$ & $13,2: 1$ \\
\hline 20.-21. III. & 748 & 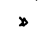 & 11,77 & 0,2467 & 0,0163 , & $47,7: 1$ & $15,1: 1$ \\
\hline Hittelwerthe: & 770 & $\operatorname{ccm}$. & 11,98 & 0,2220 & $0,0166 \mathrm{~g}$ & $53,7: 1$ & $13 ; 5: 1$ \\
\hline
\end{tabular}

Tabelle II.

\begin{tabular}{|c|c|c|c|c|c|c|c|c|}
\hline Tag & \multicolumn{2}{|c|}{$\begin{array}{l}\text { Harn- } \\
\text { menge }\end{array}$} & Ges. $-\mathrm{N}$ & $\overline{\mathrm{U}} .-\mathrm{N}$ & B. $-\mathrm{N}$ & $\frac{\text { Ges.-N }}{\overline{\mathrm{U}} .-\mathrm{N}}$ & $\frac{\overline{\mathrm{U}} \cdot-\mathrm{N}}{\mathrm{B} . \mathrm{N}}$ & Bemerkungen \\
\hline 1900 & & & & & & & & \\
\hline 21.-22. III. & 1010 & ccm. & $13,07 \mathrm{~g}$ & 0,2185 & g 0,0221 & g $59,8: 1$ & $9,9: 1$ & \\
\hline 22.-23. III. & 675 & > & 11,64 & 0,3002 & 0,0205 & $58,1: 1$ & $9,8: 1$ & \\
\hline 23.-24. III. & 690 & $>$ & 11,87 . & 0,2061 & 0,0206 & $>57,6: 1$ & $10,0: 1$ & tein \\
\hline 24.-25 III. & 720 & $\triangleright$ & 11,48 . & 0,2049 & $\times 0,0209$ & $\triangle 56,0: 1$ & $9,8: 1$ & \\
\hline 25.-26. III. & 735 & , & 10,29 , & 0,1924 & $\diamond 0,0229$ & $>53,5: 1$ & $8,4: 1$ & \\
\hline 26.-27. III. & 610 & . & 11,55 . & 0,2212 & 0,0216 & $52,2: 1$ & $10,2: 1$ & \\
\hline 27.-28. III. & 775 & > & 10,86 , & 0,2304 & $>0,0212$ & $\rightarrow 47,1: 1$ & $10,9: 1$ & \\
\hline 28.-29. III. & 660 & > & 12,01 . & 0,2233 & 0,0261 & $\$ 53,8: 1$ & $8,6: 1$ & n \\
\hline 29.-30. III. & 820 & > & 13,76 , & 0,2317 & $>0,0240$ & $>59,4: 1$ & $9,6: 1$ & \\
\hline 30.-31. III. & 960 & $>$ & 13,77 . & 0,2142 & $\gg 0,0272$ & . $64,4: 1$ & $7,9: 1$ & \\
\hline 31. III.-1. IV. & 650 & $\triangleright$ & 11,85 . & 0,2085 & $>0,0164$ & $\gg 56,8: 1$ & $12,7: 1$ & \\
\hline 1.-2. IV. & 915 & $\triangleright$ & 13,37 . & 0,2557 & $\$ 0,0195$ & $\times 52,3: 1$ & $13,1: 1$ & kein \\
\hline 2.-3. IV. & 698 & , & 12,83 , & 0,2106 & $\gg 0,0175$ & $>60,9: 1$ & $12,1: 1$ & Caffein \\
\hline 3.-4. IV. & 835 & $\triangleright$ & 14,52。 & 0,2500 & $(1,0192$ & $>58,1: 1$ & $13,0: 1$ & \\
\hline 4.-5. IV. & 795 & $\triangleright$ & 14,08 . & 0,2390 & $.0,0285$ & $\diamond 58,9: 1$ & $8,4: 1$ & $0,2 \mathrm{~g}$ Caffein \\
\hline 5.-6. IV. & 810 & $\triangleright$ & 14,94 , & 0,2307 & 0,0279 & $.64,8: 1$ & $8,3: 1$ & täglich \\
\hline
\end{tabular}


Nach Beendigung der ersten Versuchsreihe erhielt der Patient an 4 Tagen je 0,05 g Caffein, dann unmittelbar darauf an 6 Tagen je 2 mal $0,05 \mathrm{~g}$ Caffein. Alsdann folgte eine Periode von 4 Tagen mit Ausschluss des Diureticums, und schliesslich eine solche von 2 Tagen, an welchen der Patient täglich $2 \mathrm{mal}$ $0,1 \mathrm{~g}$ des Medicamentes erhielt.

Auch bei diesen Versuchsreihen wurden stets Doppelanalysen ausgeführt, deren Mittelwerthe in Tabelle II, Seite 107, angegeben sind.

Die Resultate ergeben sich ohne Weiteres bei Durchsicht der Tabelle III, welche die Mitlelzahlen der 5 Versuchsperioden enthält.

Tabelle III.

Mittelzahlen der Caffein-Versuchsperioden.

\begin{tabular}{|c|c|c|c|c|c|c|c|}
\hline Tage & $\begin{array}{l}\text { Harn- } \\
\text { menge }\end{array}$ & Ges.-N & $\overline{\mathrm{U}} .-\mathrm{N}$ & B. $\cdot \mathrm{N}$ & $\frac{\text { Ges.-N }}{\overline{\mathrm{U}} .-\mathrm{N}}$ & $\frac{\overline{\mathrm{U}} \cdot-\mathrm{N}}{\mathrm{B} . \mathrm{N}}$ & Bemerkungen \\
\hline 1.-13. Tag & $770 \mathrm{ccm}$. & $11,98 \mathrm{~g}$ & 0,2220 & g 0,0166 & g $53,7: 1$ & $13,5: 1$ & koin Caffein \\
\hline 14.-17. & 774 & 12,01 & 0,2074 & 0,0210 & $57,9: 1$ & $9,9: 1$ & $0,05 \mathrm{~g}$ Oaffein täglich \\
\hline 18.-23. & 759 & $12,04 \times$ & 0,2188 & $>0,0240$ & $>55,5: 1$ & $9,3: 1$ & $0,1 \mathrm{~g}$ Oafioin täglich \\
\hline 24.-27. & 774 & 13,14 & 0,2312 & 0,0181 & $\star 57,0: 1$ & $12,7: 1$ & kein Caffiein \\
\hline 28.-29. & 802 & $14,51 »$ & 0,2348 & × 0,0282 & $>61,8: 1$ & $8,35: 1$ & $0,2 \mathrm{~g}$ Oaffein täglich \\
\hline
\end{tabular}

Was zunächst die Abhängigkeit der Harnsäureausscheidung von der Caffeineinnahme betrifft, so zeigt sich in Uebereinstimmung mit den Angaben von Schutzkwer und Minkowsky, dass das Caffein keine Vermehrung der Harnsäureausfuhr bewirkt. Beim Uebergang der Vorperiode zur ersten Caffeinperiode ist sogar Harnsäure- $\mathrm{N}$ von $0,222 \mathrm{~g}$ auf $0,2074 \mathrm{~g}$ zurückgegangen, um in der zweiten, sechs Tage dauernden Caffeinperiode sich dem normalen Werthe um wenige Milligramme zu nähern. Die geringe Zunahme an Harnsäure-N, $3,6 \mathrm{mg}$, welche in der letzten Caffeinperiode gegenüber der vorhergehenden normalen Periode zur Ausscheidung gelangt ist, ist zweifellos nicht auf das Caffein zurückzuführen, sondern steht im Zusammenhang mit dem Plus an Gesammtstickstoff. Dagegen vermehrt das Caffein in deutlicher Weise die 
Purinbasen des Harnes. Nach Eingabe von nur $0,050 \mathrm{~g}$ Caffein steigt der Stickstoff derselben von $0,0166 \mathrm{~g}$ auf $0,0210 \mathrm{~g}$, nach Eingabe von $0,1 \mathrm{~g}$ Caffein auf $0,024 \mathrm{~g}$ an. Eine Dosis von $0,2 \mathrm{~g}$ Caffein treibt den Purinbasen-N von $0,0181 \mathrm{~g}$ auf $0,0282 \mathrm{~g}$.

Rechnet man die Zunahme an Purinbasen- $\mathrm{N}$ in Procenten vom eingegebenen Caffeinstickstoff um, so ergibt sich, dass

1. bei Eingabe von $0,050 \mathrm{~g}$ Caffein $33,3 \%$ seines Stickstoffes

$2 .>>0.100>, 28,0 \%$ >

$3 .>>0,200>>19,3 \%$ > >

als Purinbasenstickstoff im Harne ausgeschieden werden.

Somit ist die Zunahme an Basenstickstoff nicht proportional der eingeführten Menge an Caffein, sondern mit steigenden Dosen des letzteren nimmt die procentische Umwandlung in niedere Homologe des Xanthins ab.

Wie stehen diese Resultate im Einklang mit den Angaben von Burian und Schur, sowie von Albanese? Burian und Schur haben während drei Tagen den Aufguss von $160 \mathrm{~g}$ Kaffee, welcher 1,0-1,2 g Caffein enthält, getrunken und danach eine Steigerung des Purinbasen- $\mathrm{N}$ gefunden, welche $35-40 \%$ des eingeführten Caffein- $\mathrm{N}$ entspricht. Diese Zahlen übertreffen die nach unseren Resultaten zu erwartenden Werthe bedeutend. Doch dürften unsere Angaben nicht ohne Weiteres mit denen der genannten Autoren $z u$ vergleichen sein, weil letztere, wie erwähnt, Kaffeeaufguss genommen, wir dagegen reines Caffein gegeben haben.

Bei dem Versuche von Albanese wurden die Purinbasen nach Einnahme von $2 \mathrm{~g}$ Caffein innerhalb drei Tagen um $0,155 \mathrm{~g}$ vermehrt. Da die nach Caffein auftretenden Purinbasen aller Wahrscheinlichkeit nach auch beim Menschen, wie beim Hunde und Kaninchen, ${ }^{1}$ ) aus einem Gemenge von Dimethylxanthin und Monomethylxanthinen bestehen werden, so wird denselben ein Stickstoffgehalt von etwa 32,4\% (Mittel von Dimethylxanthin und Monomethylxanthin) zukommen müssen. Hieraus berechnet sich, dass $9-10 \%$ des Caffein- $\mathrm{N}$ beim Albanese-

1) M. Krüger, Bericht der deutschen chem. Gesellsch., Bd. XXXII, S. 2818 und 3336 . 
schen Versuche in Purinbasen- $\mathrm{N}$ übergegangen sind, was mit unseren Resultaten im Einklang steht.

Die Unabhängigkeit der Harnsäureexcretion und das Anwachsen der Purinbasen nach Caffeinzufuhr kommt in schöner Weise in dem Absinken des Verhältnisses von Harnsäure-N zu Basen-N zum Ausdruck. Dasselbe beträgt normal 13,5:1 resp. 12,7:1 (siehe die 2. normale Periode) und fällt ab auf 9,9:1, 9,3:1 und schliesslich nach Eingabe von $0,2 \mathrm{~g}$ Caffein auf $8,35: 1$.

Von weit grösserem Einfluss auf die Basenausscheidung mit dem Harne als das Caffein erweist sich das Theobromin. Derselbe Patient erhielt nach 3tägiger Pause, während welcher die Erscheinungen der Caffeinverabreichung verschwinden mussten, am 9. und 10. April je 0,4 g Theobromin.

Tabelle IV.

Versuch mit Theobromin.

\begin{tabular}{|c|c|c|c|c|c|c|c|}
\hline Tag & $\begin{array}{l}\text { Harn- } \\
\text { menge }\end{array}$ & Ges.-N & $\overline{\mathrm{U}} \cdot \mathrm{N}$ & B. $-\mathrm{N}$ & $\frac{\text { Ges.-N }}{\bar{U} .-\mathrm{N}}$ & $\frac{\overline{\mathrm{U}} .-\mathrm{N}}{\mathrm{B} . \mathrm{N}}$ & Bemerkungen \\
\hline $\begin{array}{c}1900 \\
9 .-10 . \text { IV. } \\
\text { 10.-11. IV. }\end{array}$ & $\begin{array}{l}660 \mathrm{ccm} . \\
815\end{array}$ & $\left|\begin{array}{l}10,79 \mathrm{~g} \\
12,95\end{array}\right|$ & $\begin{array}{l}0,1848 \\
0,1927\end{array}$ & \begin{tabular}{l|l}
$\mathrm{g}$ & 0,0642 \\
. & 0,0858
\end{tabular} & \begin{tabular}{l|l}
$\mathrm{g}$ & $\tilde{5} 8,4: 1$ \\
& $67,0: 1$
\end{tabular} & $\begin{array}{ll}2,9 & : 1 \\
2,25: 1\end{array}$ & $\left\{\begin{array}{c}0,4 \mathrm{~g} \text { Theobromin } \\
\text { tåglich }\end{array}\right.$ \\
\hline Mittelwerthe: & $738 \mathrm{ccm}$. & $11,87 \mathrm{~g}$ & 0,1890 & g 0,0750 & $\mathrm{~g} 62,7: 1$ & $2,6: 1$ & \\
\hline
\end{tabular}

Gleich dem Caffein vermehrt Theobromin die Harnsäureexcretion nicht. Der Harnsäure-N beträgt nur $0,1890 \mathrm{~g}$, ist also noch unter dem Harnsäurewerth der ersten caffeinfreien Periode, welche den gleichen Gesammt-N-Werth zeigt, herabgegangen. Dagegen ist der Basenstickstoff gewaltig gestiegen, am ersten Tage auf $0,0642 \mathrm{~g}$, am zweiten auf $0,0858 \mathrm{~g}$; zweifellos wird eine Vermehrung auch noch am dritten Tage - ohne weitere Zufuhr von Theobromin - wahrzunehmen sein. Entsprechend dem Anwachsen des Purinbasen-N ist das Verhältniss von Harnsäure- $\mathrm{N}$ zu Purinbasen-N bis auf 2,6:1 im Mittel gefallen, d. h. der Basenstickstoff beträgt mehr als den dritten Theil des Harnsäurestickstoffes. Nicht weniger als $47 \%$ des eingeführten Theobromin-N erscheint im Harne als Purinbasen-N wieder. 